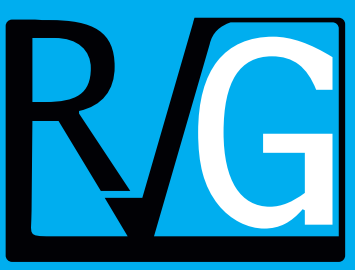

Año 21 No. 74

Abril - Junio 2016

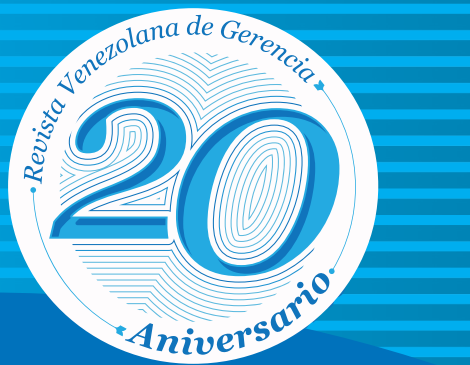

Venezolana de
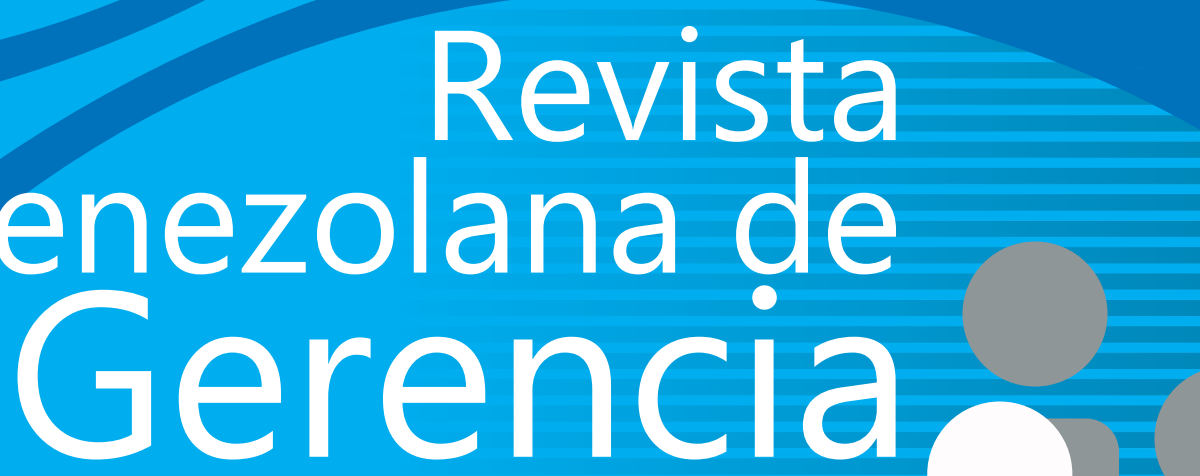


\title{
Formación profesional y procesos de innovación a nivel regional*
}

\author{
Brunet Icart, Ignasi ${ }^{1}$ \\ Mara, Liviu Catalin²
}

\begin{abstract}
Resumen
Respecto al modelo formativo que debe acompañar las apremiantes transformaciones que requiere el actual modelo económico se señala el fomento de la innovación, si bien constriñéndola a la relación entre universidad/empresa. Una combinación ineludible, sin duda, pero que obvia otra relación, la que se establece entre Centros de Formación Profesional y pymes. El objetivo de este artículo es explicar esta última relación apoyándose en la teoría de los sistemas regionales de innovación. La metodología que se ha utilizado ha sido el análisis comparado de las propuestas y perspectivas sobre sistemas, modos de innovación y formación profesional a través del análisis e interpretación de textos. En este artículo, como conclusión, se resalta que los procesos de innovación que impactan positivamente en el nivel de competitividad y en la tasa de crecimiento no son generados exclusivamente por instituciones de innovación y desarrollo sino que también los Centros de formación profesional pueden realizar una importante contribución a la innovación en las pymes.
\end{abstract}

Palabras clave: modos de innovación; sistemas de innovación; formación profesional; pymes.

Recibido: 10.04.16 Aceptado: 30.05.16

$1 \quad$ Este artículo es un resultado del proyecto de investigación "Formación Profesional y pymes: el reto de la innovación en Cataluña", financiado por RecerCaixa, con el número de referencia: 2014ACUP0077. *El investigador principal de este proyecto es el Dr. Ignasi Brunet Icart. Catedrático de Universidad, Departamento de Gestión de Empresas. Universitat Rovira i Virgili. Correo electrónico: ignasi.brunet@urv.cat

2 Personal docente e investigador, Departamento de Gestión de Empresas. Universitat Rovira i Virgili. Correo electrónico: liviucatalin.mara@urv.cat 


\title{
Vocational training and innovation processes at the regional level
}

\begin{abstract}
Regarding the training model that should accompany the pressing transformations required by the current economic model it is mentioned fostering innovation, although it is limited to the relationship between university/enterprise. An unavoidable combination, without doubt, but which ignores another relationship, the one that is established between Vocational Training Centres and the SMEs. The aim of this article is to explain this last relationship relying on the theory of regional innovation systems. The methodology used was the comparative analysis of the proposals and perspectives on systems, modes of innovation and vocational education and training through the analysis and interpretation of texts. In the conclusion of this article, it is pointed out that innovation processes that positively impact the level of competitiveness and growth rate are not generated exclusively by institutions from the R\&D system, whereas Vocational Training Centres can also make an important contribution to the innovation in SMEs.
\end{abstract}

Keywords: modes of innovation; innovation systems; vocational training; SMEs.

\section{Introducción}

Con el fin del ciclo keynesianofordista se ha ido priorizando los factores que están agrupados bajo la etiqueta de creatividad y aprendizaje tecnológico, y entre éstos, la creación de nuevos conocimientos, dado que el conocimiento se ha transformado en la base fundamental de la vida económica y social en el capitalismo contemporáneo. En este capitalismo, el conocimiento, en sus diversas formas simbólicas, estéticas, informativas, entre otras, se ha presentado, en los países del núcleo duro o core del capitalismo como el insumo más valioso para la producción del beneficio y por ende la acumulación.

Este mensaje, ha sido uno de los que más espacio de maniobra ha tenido en las últimas décadas. De hecho, la constante creación de productos financieros ha sido vista como un proceso innovador y creativo, y por tanto como aspectos dinámicos y positivos de unos mercados pretendidamente eficientes (MacKenzie et al, 2007).

El interés por la innovación, la cual ha sido definida en términos de "cómo hacer algo nuevo o hacer las cosas que existen en una nueva forma" (Guthrie et al, 2004:10), se apoya en la convicción de que la emergencia histórica de una economía del conocimiento globalizada (Lundvall et al, 2008) tiene que ver con que esta economía está dando mayor importancia que antes a los factores que puedan estar agrupados bajo la etiqueta de aprendizaje, al transformarse éste en una base fundamental de la vida económica y social (Stiglitz et al, 2015).

En este sentido, la importancia de la innovación describe las tendencias de las economías, afectadas por el ritmo de los avances científico-tecnológicos, su creciente complejidad, así como su papel determinante en los engranajes de la globalización económica, que está alterando la lógica de actuación de individuos, empresas, ciudades, 
redes de intervención público-privada, regiones y países.

Este artículo expone que respecto al modelo formativo que debe acompañar a las apremiantes transformaciones que requiere el actual modelo económico se señala el fomento de la innovación, si bien constriñéndola a la relación entre universidad/empresa. Una combinación ineludible, sin duda, pero que obvia otra relación, la que se establece entre Centros de Formación Profesional (CFP) y pymes. El objetivo de este artículo es explicar esta última relación apoyándose en la teoría de los sistemas regionales de innovación (SRI).

La metodología que se ha utilizado ha sido el análisis comparado de las propuestas y perspectivas sobre sistemas de innovación (SI) a través del análisis e interpretación de textos. Se pretende, a través del análisis de diversos autores seleccionados, dar cuenta de una línea de investigación convergente en relación a la cuestión de la teoría de los SRI. Esta teoría se apoya en estudios recientes de innovación en economía y sociología (epígrafe 2) y en los estudios sobre sistemas de innovación (epígrafe 3). Estudios que enfatizan tanto la naturaleza no lineal de la innovación como la naturaleza social del cambio técnico. Posteriormente, se desarrollan los estudios que destacan la relación entre la innovación y la FP.

\section{Innovación en economía y en sociología}

Los estudios de la innovación en la ciencia económica han institucionalizado una fuerte sub-disciplina: la economía de la innovación, que desde dinámicas de "colegio invisible" ha ido ganando posiciones respecto a la mayoritaria perspectiva disciplinar de la economía neoclásica (Hall et al, 2010). Existen dos perspectivas económicas que han dado una mayor atención a la innovación: el enfoque evolutivo y la economía aplicada. El autor clásico en la ciencia económica de la innovación, e inspirador del enfoque evolutivo, ha sido sin duda Schumpeter (2012). Para este autor, el emprendedor es el agente económico que innova, el que proporciona la respuesta creativa del sistema económico, al constituir la innovación la fuerza más importante del desarrollo económico, proceso denominado como "destrucción creadora".

En la concepción schumpeteriana, el encargado de introducir nuevos inventos en la actividad productiva, es el emprendedor que destruye, mediante su innovación, la posición inicial de mercado $\mathrm{y}$, por ello, consigue cierta posición temporal de dominio en el mercado en el que actúa. En esta concepción, las innovaciones surgen mayormente en el interior de las grandes empresas debido a su actividad investigadora, cada vez más impulsada por especialistas entrenados dentro de éstas que innovan de acuerdo a los requerimientos de las mismas.

Esta concepción de la innovación es una concepción orientada por la oferta, al constituir un proceso lineal de búsqueda consciente del conocimiento que va desde la investigación básica hasta el desarrollo tecnológico basado sobre un carácter esporádico, siendo un acontecimiento singular que se prolonga en el tiempo, y que se desarrolla en un determinado tipo de estructura empresarial.

En el enfoque evolucionista la concepción de la innovación se hace más compleja, cubriendo aspectos que van desde la influencia de la tecnología en la macroeconomía hasta el estudio de las trayectorias o senderos tecnológicos, pasando por los estudios comparados de 
SI (Nelson, 1993). Desde este enfoque, las innovaciones tecnológicas dependen de que las empresas no exploran un stock de conocimiento libre sino que su proceso de búsqueda es un proceso de mejora y de diversificación a partir de su propia base de conocimiento, de tal forma que sus posibilidades dependen de lo que han hecho en el pasado, es decir, de sus propias rutinas organizativas.

Se establece, así, que la empresa se caracteriza por una serie de hábitos que han sido seleccionados por la competencia en los mercados, y que se aplica a la variación o innovación de dichas prácticas como consecuencia de su operar adaptativo respecto al entorno competitivo. Así, cuando se produce un cambio en el mercado, las empresas se adaptan mediante la variación de sus rutinas y el entorno selecciona a aquéllas que mejor se ajustan a él. Las rutinas más exitosas son aquellas que sobreviven a la selección de entorno. La retención de estas prácticas completa el propio proceso evolutivo de la empresa.

La innovación no sucede, por tanto, sólo a través de rupturas o saltos verticales, también avanza, generalmente, de forma continuada, dentro de la trayectoria seguida por el proceso de producción, siendo este el lugar donde se introducen mejoras del producto y del proceso. Las opciones técnicas a las que se enfrenta una empresa no conforman un menú determinado exógenamente, tal y como indica el enfoque estándar o neoclásico. Más bien son idiosincráticas, en la medida en que resultan de su propia trayectoria y, específicamente, de los logros y procesos de sus acciones de búsqueda, al ejercitar rutinas específicamente orientadas a este fin. Lo que explica que la empresa dependa del patrón de actividades productivas que ha sido capaz de seguir en el pasado.

Patrón de actividades que genera una historia de aprendizaje que no se limita tan sólo a la obtención de un mayor acceso a la información, sino también a la capacidad para adquirir y valorar tanto las formas de conocimiento codificadas como las tácitas, es decir, la capacidad para aprender y adaptarse ante circunstancias competitivas rápidamente cambiantes. Surge así la idea de que las empresas compiten, principalmente o en primera instancia, a través de la tecnología, considerando a ésta no como una variable externa que le viene impuesta a la empresa, sino principalmente una variable interna, endógena, y definiéndola no como información, sino como conocimiento (Brunet et al, 2010).

Para Baumol (2005) la visión schumpeteriana de la innovación orientada por la oferta está tan influida por un entorno institucional, financiero y tecnológico como alejada del actual entorno de integración comercial, apertura de mercados financieros y cambio técnico y organizativo acelerado. En este nuevo entorno, el motor del capitalismo, y de la prosperidad que conlleva, se debe a una doble fuente interna y externa a la empresa, es decir, a la combinación de una intensa actividad innovadora sistemática dentro de la empresa y de la colaboración inter-empresarial en la creación y uso de innovaciones.

En este sentido, Baumol elabora una concepción de la innovación orientada desde el mercado y definida como un proceso permanente, recurrente y continuo, vinculado a entornos competitivos, de economía globalizada, que impulsa la aparición de nuevas formas de organización. Su unidad de análisis y referencia son las grandes empresas norteamericanas. En ellas Baumol observó cómo fueron reduciéndose su dimensión externalizando funciones, buscando la eficiencia tanto en la desintegración vertical como en la flexibilidad, lo cual 
liberó recursos para dedicarlos a la estrategia de innovación.

Esta estrategia requiere sustituir la lógica de la oferta por los incentivos del mercado y por la demanda tecnológica proveniente del sector productivo. De este modo, esta lógica pasa a convertirse en una prioridad para definir los criterios de política tecnológica e innovación.

Hasta los años 80, aproximadamente, la sociología de la innovación ni cuestionó la validez de la tecnología, ni criticó la extendida creencia del determinismo tecnológico, aquella basada en suponer que la tecnología, debido a su naturaleza técnica $y$, por ende, al conocimiento incorporado en todo artefacto tecnológico, se constituía en una ciencia aplicada claramente imparcial, neutral y objetiva, siendo tan sólo los conocimientos y las tecnologías correctas, por efectivas y adecuadas, las que se desarrollan y aplican.

Desde la década de 1980 esta creencia empezó a ser cuestionada por diferentes perspectivas constructivistas, por ejemplo MacKenzie et al, (1984) y Bijker et al, (1987). Para el constructivismo social la tecnología es una construcción social, resultado de un proceso social de innovación, lo que vino a complementar el análisis de los efectos sociales de la aplicación tecnológica. Pero también dentro del constructivismo ha habido una serie de debates en torno a dos ideas básicas.

En primer lugar, profundizar en la crítica a la visión lineal de la innovación. La diferencia de tiempo entre los avances científicos y sus aplicaciones tecnológicas puede variar en su secuencia temporal y procesual, y los descubrimientos científicos no son percibidos como la única fuente de ideas para la generación de innovaciones.

Analiza y explica la innovación técnica como el resultado de un proceso abierto y sin pre-determinaciones apriorísticas, en el que diferentes actores, con sus respectivas posiciones y capacidades, intervienen y moldean el proceso y el contenido de la innovación. En este sentido, se reconoce la importancia de los itinerarios tecnológicos alternativos o marginados por la fuerza de las estructuras paradigmáticas de la ciencia y la tecnología.

Este argumento contrapesa la idea de determinismo tecnológico, con la visión de determinismo social de la tecnología, y enfatiza la importancia de la interacción entre múltiples agentes, la doble direccionalidad entre investigación y aplicación, el carácter acumulativo de los procesos de innovación y la importancia de otras fuentes de acceso a la innovación al margen de la I+D. La innovación es una actividad emergente e interactiva, que no tiene necesariamente que tener un origen 0 una fuente tecnológica (Akrich et al, 2002).

En segundo lugar, destacar la importancia de los factores sociales, culturales y de poder tanto a nivel micro como macro. Estudios cualitativos de procesos de innovación han echado luz a una variedad de aspectos que interfieren en el proceso. Se pueden destacar, por ejemplo, los estudios feministas de la tecnología que han explicitado la fuerte masculinidad de la tecnología (Faulkner, 2000).

\section{Modos de innovación}

El modelo lineal de innovación, denominado Modo 1 en la literatura científica (Jensen et al, 2007; Lundvall et al, 2010), separa investigación básica e investigación aplicada, produciendo un prototipo "en cascada" o de "goteo hacia abajo" en que el proceso de 
Formación profesional y procesos de innovación a nivel regional

Brunet Icart, Ignasi y Mara, Liviu Catalin

cambio tecnológico sigue una lógica lineal, según la cual el aumento de la investigación básica conduce a un aumento de las oportunidades para la innovación tecnológica, lo que lleva a un crecimiento del producto social.

La evidencia histórica pone en cuestión este modelo, ya que la diferencia de tiempo entre los avances científicos y sus aplicaciones tecnológicas puede variar solamente en unos meses, o bien la innovación tecnológica puede realmente preceder al descubrimiento científico o, en ocasiones, los avances científicos pueden estar basados en la invención de nuevas maquinarias y no en el sentido opuesto.

Por otra parte, el modelo lineal de innovación asume que la tecnología es información sencilla de copiar, en la medida en que las innovaciones se pueden codificar como información que se transforman en bienes y servicios cuya difusión es inmediata y barata, mediante la imitación o copia. De este modo, las innovaciones se convierten en un bien comercializable y el retraso tecnológico de un país o una empresa se puede superar mediante la compra de innovaciones.

Sin embargo, los procesos a través de los cuales surgen las innovaciones son más complejos, y tienen que ver con que en un entorno económico globalizado se requiere sustituir la lógica de la oferta por los incentivos del mercado. En este nuevo entorno, la demanda tecnológica proveniente del sector productivo pasa a convertirse en una prioridad para definir los criterios de política tecnológica e innovación (Asheim, 2010).

La complejidad de los procesos de innovación ha llevado a abogar por una evolución de la demanda social de producción de conocimientos desde un
Modo 1 hacia un Modo 2. El Modo 1 se caracteriza por la existencia de una escasa conexión entre las necesidades de la sociedad y las orientaciones de las investigaciones científicas, mientras que el Modo 2 propone que la producción de conocimientos se ha de llevar a cabo con la intención de ser aplicado para resolver las necesidades sociales (Isaksen et al, 2010).

El Modo 2 es un modo orientado al cliente (mercado o demanda) basado fundamentalmente en el desarrollo de competencias e innovaciones organizativas y que produce sobre todo innovaciones incrementales. Un modo que se caracteriza por centrarse en un conocimiento basado en la experiencia y en una continua así como pragmática recombinación de conocimientos procedentes de diversas fuentes tanto internas como externas. Así pues, implica a numerosos grupos de actores en redes y formas de organización flexibles, generando conocimiento que puede ser en gran medida tácito y especializado en su contexto de desarrollo y aplicación (es decir, dependencia del camino, de acuerdo con el enfoque evolucionista) (Johnson, 2010).

Desde el planteamiento del Modo 1 los centros de I+D han de desempeñar un rol activo en promover los cambios tecnológicos y la innovación. Esto anterior explica que en determinados áreas regionales europeas exista una tendencia creciente a fomentar estructuras de transferencia de tecnología. Áreas de desarrollo innovador, que son zonas geográficas de alta concentración de actividades innovadoras, en las que existe una elevada coordinación entre universidades, empresas y administraciones públicas, así como estructuras de intermediación como las incubadoras universitarias de empresas 
de base tecnológica y los parques científicos. En este sentido, el potencial de innovación nace de la variedad y de la colaboración (Cooke, 2004).

Desde esta percepción, emerge una visión más amplia de la innovación, de la cual se desprende la idea de que los descubrimientos científicos no son la única fuente de ideas para la generación de innovaciones, pues éstas pueden surgir de otro modo de innovación (Modo 2), y característicamente no lineal, y que se apoya sobre la noción de que a partir de las actividades productivas surgen nuevas formas de división del trabajo, que priorizan también la interacción entre múltiples agentes, resaltando el carácter acumulativo de los procesos de innovación y la importancia de otras fuentes de acceso a la innovación al margen de la I+D. Esto pone de manifiesto un fenómeno de innovación distribuida, en tanto que, la innovación también es un proceso social y territorial de carácter acumulativo e interactivo (Heijs, 2010).

De hecho, en la mayor parte de las veces, la innovación, no se realiza de forma individual sino a través de una capacidad endógena de aprendizaje e innovación colectiva. Por esto, el Modo 2 resalta la naturaleza social/institucional del aprendizaje y la innovación, esto es, los procesos innovadores y las dinámicas de aprendizaje colectivo que acaecen en regiones o localidades específicas, cuyo dinamismo y capacidad innovadora está en la presencia de un elevado conjunto de pymes industriales y de servicios a la producción que operan en red, combinando estrategias de competencia por ocupar los mismos mercados con otras de cooperación formal o informal.

Se asume, por tanto, el pensamiento institucionalista que define la economía como algo más que una colección de empresas aisladas y mercados dirigidos por las preferencias racionales y por un conjunto de reglas estándar. La economía se concibe como una composición de influencias colectivas que modelan la acción individual y como una entidad diversificada que sigue una trayectoria histórica modelizada por influencias culturales y socio-institucionales heredadas (Amin, 2008). En este sentido, las capacidades de innovación tienen efectos territoriales en tanto que el medio emerge como un elemento necesario y crucial en los procesos de innovación, siendo el resultado de sistemas o medios en los que la empresa está inserta y no del comportamiento de empresas individualmente consideradas. Estos sistemas son conceptualizados como sistemas productivos, sistemas industriales o SI.

\section{Sistemas de Innovación}

Se entiende por SI al conjunto de instituciones y agentes, tanto privados como públicos, que interactúan en la producción, difusión y utilización de nuevos conocimientos y avances tecnológicos (Brunet y Baltar, 2011). Bajo esta concepción la innovación es un proceso interactivo y sistémico, con conexiones y realimentaciones entre las distintas fases y niveles (Fageberg et al, 2005).

El sistema nunca alcanza un estado de equilibrio, puesto que los procesos evolutivos son abiertos y dependientes de una trayectoria histórica. Además, el concepto de SI pone énfasis también en que cualquier economía necesita disponer de un sistema de ciencia y tecnología adecuado a su dimensión y a sus 
capacidades, bien diseñado y protegido, y más específicamente de entidades de transferencia y difusión de ideas, cualificaciones, conocimiento e información.

Se destacan cuatro grandes desarrollos del enfoque SI: en primer lugar la perspectiva propuesta por el grupo IKE de la Universidad de Aalborg, liderado por Lundvall (2002). Para este grupo, la innovación es el resultado de un proceso de aprendizaje interactivo, en el que las relaciones entre productorusuario y el sistema de generación y distribución de conocimiento en el mercado laboral resultan de especial importancia. Asimismo, se destaca que debe aceptarse que las variaciones locales y nacionales pueden conducir a diferentes trayectorias de desarrollo y a una creciente diversidad, en vez de a la estandarización y a la convergencia. En segundo lugar en la cuestión espacial y geográfica en la innovación la dimensión sub-nacional o sub-estatal ha cobrado una alta importancia, desarrollando el concepto de SRI (Muller et al, 2008).

En una economía global los diversos países o regiones cumplen distintas funciones dentro del sistema nacional o internacional de producción e innovación. Así, existen regiones y países centrales que son el corazón del sistema nacional o internacional de innovación, y otras regiones cuyo desarrollo se basa en una función complementaria en apoyo a las regiones centrales o en la explotación de los recursos endógenos. Un sistema de innovación regionalizado fuerte es aquel que tiene vínculos sistémicos entre las fuentes de producción del conocimiento (universidades, organizaciones de investigación, CFP), intermediarios (gobierno y servicios de innovación privados) y las empresas tanto grandes como pequeñas (Gebauer et al, 2005).
En tercer lugar la dimensión sectorial ha recibido una creciente atención, y provee al enfoque SI de una incipiente formalización sobre los procesos evolutivos. Para Malerba (2005), el enfoque del Sistema Sectorial de Innovación (SSI) se fundamenta en tres pilares analíticos: el conocimiento y la tecnología, los actores y las redes, y las instituciones. Este enfoque muestra una tendencia al estudio de sectores de tecnología media y alta, pero se puede decir que el SSI ha hecho dos contribuciones importantes.

Por un lado, a nivel conceptual ha resaltado la importancia de las organizaciones y empresas, así como de sus relaciones. Por otro lado, ha destacado la relevancia del conocimiento y la tecnología, aunque a veces parece caer en un cierto determinismo tecnológico al enfatizar el cambio tecnológico como factor determinante del cambio en los actores organizativos y en las relaciones entre ellos, ya que ha enfatizado la importancia de los tipos de conocimiento y tecnología en la innovación sectorial.

En cuarto lugar un desarrollo reciente ha puesto el énfasis en la importancia del aprendizaje y de la generación de competencias y habilidades al nivel organizativo, interorganizativo o sectorial, y general en los sistemas educativos y de formación. En este sentido, algunos estudios han llamado la atención sobre la importancia de la relación entre el sistema de educación y formación y el SI (Descy et al, 2005). Estos estudios apuntan a que los SI tienen su origen en los sistemas de producción y en los sistemas de desarrollo de recursos humanos.

Los sistemas de educación y los mercados laborales están nacionalmente y regionalmente constituidos y juegan 
un papel clave en la creación de competencias y en la conformación de las bases de los procesos de innovación. Por ello, Edquist (2005) sugiere que el enfoque SI debería añadir una tercera dimensión, el citado sistema de educación y formación, especialmente a través de la noción de construcción de habilidades o competencias.

Estos cuatro grandes desarrollos del enfoque SI coinciden con la cada vez más aceptada diferenciación de dos grandes tipos o modos de innovación: la visión estrecha del tipo ScienceTechnology-Innovation (STI) y la visión amplia del tipo Doing-Using-Interacting (DUI). Mientras el STI tiende a mantener cierta importancia de la visión linear y formal de la innovación, el DUI enfatiza los procesos de abajo-arriba no-lineares, informales y multidireccionales de aprendizaje y flujos de conocimiento, en proximidad al sistema productivo. Así pues, mientras el STI prioriza el desarrollo de conocimiento desde la investigación, la educación superior, la gran empresa, etc., acompañada de jerarquías formales y fuertes regulaciones (propiedad intelectual, patentes, etc.), el DUI prioriza el aprendizaje desde las pymes, el sistema educativo y la FP, mediante regulaciones blandas y relaciones interactivas.

En clave macro-sociológica, mientras el STI es visto como un instrumento de generación de ciertas élites de investigación, el DUI opta por la distribución y difusión del conocimiento tanto en amplios espectros de la sociedad como en la organización del trabajo. Estas diferencias entre los dos modelos quedan reflejadas en el siguiente cuadro 1.

\section{Cuadro 1 \\ Principales diferencias entre los Modos STI y DUI}

\begin{tabular}{|c|c|c|c|}
\hline \multirow[t]{2}{*}{ Nivel } & Dimensión & Modelo STI & Modelo DUI \\
\hline & Procesos & $\begin{array}{l}\text { - Procesos de aprendizaje arriba-abajo } \\
\text { - Procesos formales } \\
\text { - Procesos unidireccionales }\end{array}$ & $\begin{array}{l}\text { - Procesos de aprendizaje abajo-arriba } \\
\text { - Procesos informales } \\
\text { - Procesos multidireccionales }\end{array}$ \\
\hline
\end{tabular}

Microsocial

Aprendizaje - Aprendizaje formal: educación

superior, investigación
- Aprendizaje informal: interactivo, práctico, intervención múiltiples agentes

\footnotetext{
Macrosocial

Procesos - Generación de élites de investigación

- Difusión y distribución del conocimiento

Aprendizaje - Compra-venta conocimiento

- Spillover, acceso abierto
}

Fuente: Elaboración propia. 
Formación profesional y procesos de innovación a nivel regional

Brunet Icart, Ignasi y Mara, Liviu Catalin

\section{Formación profesional, innovación y pymes}

A partir de la diferenciación de estos dos modos de innovación, en los últimos años, gran parte del debate sobre innovación se ha centrado en estudiar los tipos de flujos de conocimiento que pueden ayudar a las pymes y a las grandes empresas (sobre todo las primeras) a absorber nuevos inputs y a transformarlos en capacidades relevantes. En este sentido, diferentes análisis han demostrado que en diferentes países y sistemas productivos hay diversos perfiles organizativos con diferentes modelos de innovación.

Por ejemplo, hay países que adoptan el planteamiento de base científico/tecnológica que ha predominado en los estudios sobre innovación durante décadas, considerando como principales inputs para las actividades de innovación indicadores como el gasto en I+D y el capital humano (por ejemplo, cifra de graduados en ciencia y tecnología). Así, los modos más tradicionales de innovación de países como EEUU, Japón - Suecia se basan en considerables inversiones en I+D y capital humano. Sin embargo, también destaca la capacidad de economías como la danesa y la italiana para el desarrollo de innovaciones (incrementales) y obtener un desempeño y un desarrollo económico y social mejores con menores niveles de inversión en actividades de conocimiento formal (actividades como gasto en I+D, infraestructuras de conocimiento, capital humano) (Parrilli et al, 2010).

Estos hallazgos han hecho necesario abrir la "caja negra" de la innovación e identificar los factores de innovación que se centran menos en el flujo de conocimiento explícito y le dedican más atención al flujo de conocimiento tácito que genera inputs importantes para el proceso innovador global, pero que son difícilmente medibles (Bitard et al, 2008). Entre estos aspectos de conocimiento tácito, el proceso de aprendizaje interactivo ha sido identificado y analizado con creciente interés como uno de los factores clave. Se considera que el conocimiento tácito fluye a través de las interacciones y de los procesos de aprendizaje, si bien estos últimos tienen lugar mediante la fabricación de productos y el diseño de procesos, el uso de técnicas y maquinaria, así como de la interacción con otros agentes sociales.

En línea con lo anterior, Arundel et al, (2007) identificaron cuatro modalidades de perfiles organizativos en funcionamiento en la Unión Europea, así como los modelos específicos de cada país. Se trata de: artesanía, taylorismo, producción ágil y organización que aprende. A partir de esto, Jensen et al, (2007) también identificaron los comportamientos de las empresas en relación a su manera de innovar y los agruparon en cuatro: (1) el modo de aprendizaje bajo (que de alguna manera respondía al modo organizativo tipo artesanal); (2) el modo STI; (3) el modo DUI, y (4) el modo STI-DUI o modo de innovación combinado, basado en los dos modos anteriores a la vez. En cuanto al último modo, Parrilli y Heras (2016) han contrastado la importancia del modo STI-DUI o combinado en un estudio aplicado a empresas del País Vasco (España), y han encontrado una complementariedad entre los dos modos de innovación.

Entre estas cuatro modalidades, es la última la que parece ser el mejor modo en términos de potencial de 
flujo de conocimiento, procesos de aprendizaje $\mathrm{y}$, en consecuencia, del crecimiento y desarrollo de las empresas, las regiones y los países, ya que la primera modalidad (STI) contribuye al conocimiento científico y técnico más avanzado, mientras que la segunda (DUI) añade la posibilidad de diseminar este conocimiento entre un público más amplio dentro y entre las organizaciones, y de ayudar a sus trabajadores a absorberlo de una forma eficaz y productiva.

El modo de innovación combinado resalta la necesidad de que las regiones construyan ventajas competitivas basándose en estrategias de innovación que deben ser fruto de un proceso participativo, en el que las organizaciones de conocimiento, entre las que cabe incluir a los CFP resultan actores clave (Ranga et al, 2008).

Estos centros pueden contribuir al diseño de estrategias de innovación con su conocimiento de tecnologías y mercados, con las conexiones y redes que con frecuencia poseen con agentes de conocimiento externos al entorno local y con la imagen de relativa neutralidad con que generalmente son vistos por los restantes agentes. $Y$ la necesidad de contar con ellos se hace todavía más palmaria en regiones en que se carece de infraestructuras de I+D (universidad, centros tecnológicos y similares).

Algunos estudios dentro de la perspectiva de los SRI han puesto de manifiesto la especificidad de las capacidades de innovación de las Pymes, la importancia de los procesos de aprendizaje interactivo entre empresas y las dificultades en las relaciones con los agentes de I+D (Asheim et al, 2005).

También se llama la atención sobre la importancia de la relación entre el sistema de formación y el sistema de innovación, señalándose que los obstáculos para mejorar la capacidad innovadora de las empresas no se sitúan en los bajos niveles de gastos en $I+D$, sino en la presencia generalizada de unos entornos de trabajo que son incapaces de proporcionar un ambiente fértil para la innovación (Brunet et al, 2015).

Lundvall et al, (2008) señalan que uno de los factores que impide el desarrollo de las capacidades innovadoras es los bajos niveles de inversión en FP. Es más, como subraya la literatura australiana (Toner, 2009), desde una definición de roles en la generación de conocimiento complementaria de la que llevan a cabo la universidad y los centros tecnológicos, los CFP pueden cumplir un papel de puente entre las pymes y los centros de investigación y universidades.

Pero, desgraciadamente, no suele ser frecuente que se dé esa estrecha relación entre CFP y resto de infraestructuras de I+D (universidad, centros de investigación cooperativa, etc.). Por ejemplo, los CFP españoles no han seguido la estela de prestación de servicios técnicos, que, según se ha mostrado en la revisión de la literatura internacional, han seguido los CFP de países como EEUU, Canadá, Australia, Finlandia (Navarro, 2014).

La importancia del sistema de FP para la innovación ya fue resaltada por Rosenfeld (1998) en su informe para la Organización para la Cooperación y el Desarrollo Económicos (OCDE), en el que sugiere que los institutos de educación técnica $y$ de formación profesional estaban en una posición mejor que las universidades para ponerse al servicio de las pymes y para facilitar la colaboración, la conectividad y las estrategias de desarrollo regionales o locales.

Sin embargo, a menudo existen barreras que dificultan la participación de institutos de formación profesional en 
Formación profesional y procesos de innovación a nivel regional

Brunet Icart, Ignasi y Mara, Liviu Catalin

estas funciones no tradicionales, como la falta de autonomía y flexibilidad de los centros, la falta de incentivos o medidas políticas adecuadas y la falta de relaciones con los agentes de I+D, como los centros tecnológicos.

Para el caso español subraya que existe una falta de coordinación entre el sector de la educación profesional y continua, y el sistema de formación de los trabajadores, gestionada de forma mayoritaria a nivel nacional por las organizaciones empresariales y sindicales (Brunet et al, 2013, 2015).

En este sentido, y a nivel de resultados, se observa en investigaciones anteriores que se ignora lo que Rosenfeld ya apuntó en 1998: que los CFP son instituciones de carácter regional o local, y que a diferencia de las universidades, son organizaciones nacidas para atender las necesidades de su entorno local o regional.

Sus alumnos provienen de tal entorno y su posterior empleo, así como la prestación de servicios técnicos y de creación de empresas, tiene lugar en dicho entorno. Es más, son con frecuencia la principal infraestructura de conocimiento existente en ese entorno local. Por eso todo intento de fortalecer los sistemas regionales de innovación debe tomarlos muy en cuenta (Field et al, 2012a, 2012b).

\section{Conclusión}

En este artículo se ha resaltado como los procesos de innovación que impactan positivamente en el nivel de competitividad y en la tasa de crecimiento no son generados exclusivamente por instituciones de $I+D$, trabajando de forma aislada respecto al mercado, sino que son producidos de forma más frecuente para la solución de problemas en un contexto de aplicación y mediante la interacción de múltiples agentes. En este sentido, los estudios que hemos analizado dentro de la perspectiva de los SRI han señalado la importancia del nivel regional de los procesos de innovación de las pymes en aspectos como el intercambio de conocimiento tácito y el aprendizaje interactivo, enmarcándose bajo la perspectiva del modo de innovación DUI o Modo 2.

El Modo 2 y la perspectiva regional de la innovación resaltan que el medio territorial constituye un elemento clave en los procesos de innovación de las pymes. En estos medios territoriales se ha llamado la atención sobre la relevancia de la potenciación de la formación en innovación en los CFP, ya que los puestos intermedios técnicos cualificados por el sistema de FP realizan (o pueden realizar) una importante contribución a la innovación en las pymes como resultado de su compromiso práctico en el diseño, instalación, operación y mantenimiento de productos y procesos.

Contribución en tanto que los productos y servicios intensivos en innovación requieren de una fuerza de trabajo cualificada capaz de una rápida adaptación a los procesos de trabajo y la innovación continua de productos. La complementariedad entre la educación, la formación y la innovación parece evidente y estudios recientes muestran que las pymes industriales de los sectores de tecnología media resultan bastante innovadoras y sus procesos de innovación requieren incluir a sus trabajadores técnicos en dichos procesos de innovación. Procesos que no tan sólo son una apuesta estratégica para garantizar el futuro de las pymes, sino también una garantía de éxito para la FP. 


\section{Referencias Bibliográficas}

Akrich, Madeleine; Callon, Michel y Latour, Bruno (2002), The key to success in innovation part I: the art of interessement. International Journal of Innovation Management, Vol. 6 , No. 2, Reino Unido, Science Policy Research Unit, University of Sussex, pp 187-206.

Amin, Ash (2008), Límites y posibilidades de la Nueva Ortodoxia regionalista. En Fernández, Victor Ramiro; Amin, Ash y Vigil, José Ignacio (Comps.), Repensando el desarrollo regional: contribuciones globales para una estrategia latinoamericana, Santa $\mathrm{Fe}$, Universidad Nacional de Litoral y Miño Dávila Editores.

Arundel, Anthony; Lorenz, Edward; Lundvall, Bengt-Åke y Valeyre, Antoine (2007), How Europe's economies learn: a comparison of work organization and innovation mode for the EU-15. Industrial and Corporate Change, Vol. 16, No. 6, Reino Unido, Oxford University Press, pp 1175-1210.

Asheim, Bjørn (2010), Nueva política regional de innovación: cómo combinar un enfoque científico con un enfoque orientado al usuario. En Parrilli, Mario Davide (Coord.), Innovación y aprendizaje: lecciones para el diseño de políticas ( $p p$ 102-114), Zamudio, Innobasque.

Asheim, Bjørn y Gertler, Meric (2005), The Geography of Innovation: Regional Innovation Systems. En Fageberg, Jan; Mowery, David y Nelson, Richard (Eds.), The Oxford Handbook of Innovation ( $\mathrm{pp}$ 291-317), Oxford, Oxford University Press.

Baumol, William (2005), The free-market innovation machine: analyzing the growth miracle of capitalism. Princeton, Princeton University Press.

Bijker, Wiebe; Hughes, Thomas y Pinch, Trevor (Eds.), (1987), The Social Construction of Technological Systems: New Directions in the Sociology and History of Technology. Cambridge, M.I.T. Press.
Bitard Pierre; Edquist Charles; Hommen Leif y Rickne Annika (2008), The paradox of high R\&D input and low innovation output: Sweden. Circle Working Papers, no.14, Sweden: Lund University.

Brunet, Ignasi y Baltar, Fabiola (2010), Desarrollo endógeno, calidad institucional e innovación. Una revisión de la teoría y de algunos de sus límites. Revista CLAD, No. 48, Venezuela, Centro Latinoamericano de Administración para el Desarrollo, pp 115-148.

Brunet, Ignasi y Baltar, Fabiola (Eds.), (2011), Creación de empresas. Innovación e instituciones. Madrid, Editorial RaMa.

Brunet, Ignasi y Rodríguez, Joan (2013), Cataluña. En Olazaran, Mikel y Brunet, Ignasi (Coords.), Entorno regional y formación profesional: los casos de Asturias, Aragón, Cataluña, Navarra y País Vasco (pp 87-128). Tarragona/País Vasco, Publicaciones de la URV/UPV-EHU Servicio de Publicaciones.

Brunet, Ignasi y Rodríguez, Joan (2015), Territorio, industria e innovación. Los casos de Cataluña y Aragón. Valencia, Tirant Humanidades.

Cooke, Philip; Heidenreich, Martin y Braczyk, Hans (2004), Regional Innovation Systems. London, Routledge.

Descy, Pascaline y Tessaring, Manfred (2005), The value of learning: evaluation and impact education and training: third report on vocational training research in Europe. Louxembourg, EUR-OP.

Edquist, Harald (2005), The Swedish ICT miracle-myth or reality? Information Economics and Policy, Vol. 17, No. 3, Estados Unidos de América, CUNY, pp 275-301.

Fageberg, Jan; Mowery, David y Nelson, Richard (Eds.), (2005), The Oxford Handbook of Innovation. Oxford, Oxford University Press.

Faulkner, Wendy (2000), The power and the pleasure? A research agenda for "making gender stick" to engineers. Science, Technology \& Human 
Formación profesional y procesos de innovación a nivel regional

Brunet Icart, Ignasi y Mara, Liviu Catalin

Values, Vol, 25. No. 1, Estados

Unidos de América, Brandeis University, pp 87-119.

Field, Simon; Kis, Viktória y Kuczera, Małgorzata (2012a), Vocational education and training in Spain. En OECD, Postsecondary Vocational Education and Training Pathways and Partnerships. Paris, OECD Publishing.

Field, Simon; Kis, Viktória y Kuczera, Małgorzata (2012b), A skill beyond school commentary on Spain. OECD Reviews of Vocational Education and Training. Paris, OECD Publishing.

Gebauer, Andrea; Nam, Chang Woon y Parsche, Rüdiger (2005), Regional technology policy and factors shaping local innovation networks in small German cities 1. European Planning Studies, vol. 13, no 5, Bélgica, Association of European Schools of Planning (AESOP), pp 661-683.

Guthrie, Hugh y Dawe, Susan (2004), Overview. En Vocational education and training and innovation: Research readings (pp. 10-20). Adelaide, National Centre for Vocational Education Research Ltd, Australia.

Hall, Bronwyn y Rosenberg, Nathan (ed.), (2010), Handbook of the Economics of Innovation. Holanda, Elsevier.

Heijs, Joost (2010), Política tecnológica, aprendizaje y capacidad de absorción de conocimientos: los círculos viciosos y virtuosos. En Parrilli, Mario Davide (Coord.), Innovación y aprendizaje: lecciones para el diseño de políticas ( $p p$ 324-352). Zamudio, Innobasque.

Isaksen, Arne y Karlsen, James (2010), Modo combinado y complejo de innovación en el desarrollo de un clúster regional: el clúster de materiales ligeros de Raufoss, Noruega. En Parrilli, Mario Davide (Coord.), Innovación y aprendizaje: lecciones para el diseño de políticas ( $p p$ 268-288). Zamudio, Innobasque.

Jensen, Morten Berg; Johnson, Bjorn; Lorenz, Edward y Lundvall, Bengt-Åke (2007),
Forms of Knowledge and Modes of Innovation. Research Policy, Elsevier, Vol. 36, No. 5, pp 680-693.

Johnson, Bjorn (2010), Organización e innovación: ciudades creativas. En Parrilli, Mario Davide (Coord.), Innovación y aprendizaje: lecciones para el diseño de políticas (pp. 288-302). Zamudio, Innobasque.

Lundvall, Bengt-Åke (2002), Innovation, Growth and Social Cohesion: the Danish Model. Cheltenham, Edward Elgar.

Lundvall, Bengt-Åke; Rasmussen, Palle y Lorenz, Edward (2008), Education in the Learning Economy: a European perspective. Policy Futures in Education, Vol. 6, No. 6, New Zealand, University of Auckland, pp 681-700.

Lundvall, Bengt-Åke y Edward, Lorenz (2010), Innovación y desarrollo de competencias en la economía del aprendizaje. En Parrilli, Mario Davide (Coord.), Innovación y aprendizaje: lecciones para el diseño de políticas ( $\mathrm{pp}$ 44-102). Zamudio, Innobasque.

MacKenzie, Donald y Wacjman, Judy (Eds.), (1984), The Social Shaping of Technology. UK, Open University Press.

MacKenzie, Donald; Muniesa, Fabian y Siu, Lucia (2007), Do economists make markets? On the performativity of economics. New Jersey, Princeton University Press.

Malerba, Franco (2005), Sectoral systems. En Fageberg, Jan; Mowery, David y Nelson, Richard (Eds.), The Oxford Handbook of Innovation (pp 380406), Oxford, Oxford University Press.

Muller, Emmanuel; Doloreux, David; Heraud, Jean-Alain; Jappe, Arlette y Zenker, Andrea (2008), Regional innovation capacities in new member states: a typology. European Integration, Maastricht University, Netherlands, Vol. 30, No. 5, pp 653-669.

Navarro, Mikel (2014), El papel de los centros de formación profesional en los sistemas de innovación regionales 
y locales. La experiencia del País Vasco. Cuadernos Orkestra 2014/7, Bilbao, Universidad de Deusto.

Nelson, Richard (1993), National Innovation Systems. A Comparative Analysis. Oxford, Oxford University Press.

Parrilli, Mario Davide y Elola, Aitziber (2010), Perfiles de innovación STI - DUI y sus repercusiones para las políticas públicas de innovación. En Parrilli, Mario Davide (Coord.), Innovación y aprendizaje: lecciones para el diseño de políticas ( $p p$ 248-266). Zamudio, Innobasque.

Parrilli, Mario Davide y Heras, Henar Alcalde (2016), STI and DUI innovation modes: Scientific-technological and context-specific nuances. Research Policy, Vol. 45, No 4, Elsevier, pp 747-756.

Ranga, Liana Marina; Miedema, Joost y Jorna, Rene (2008), Enhancing the innovative capacity of small firms through triple helix interactions: challenges and opportunities. Technology Analysis \& Strategic Management, Vol. 20, No. 6, Reino Unido, University of Edinburgh, pp 697-716.

Rosenfeld, Stuart (1998), Technical Colleges, Technology Deployment, and Regional Development. Modena, OCDE.

Schumpeter, Joseph (2012), Historia del análisis económico. Barcelona, Ariel.

Stiglitz, Joseph, y Greenwald, Bruce (2015), La creación de una sociedad del aprendizaje. México, Crítica.

Toner, Phillip (2009), Workforce skills and innovation: an overview of major themes in the literature. Paris, OCDE. 


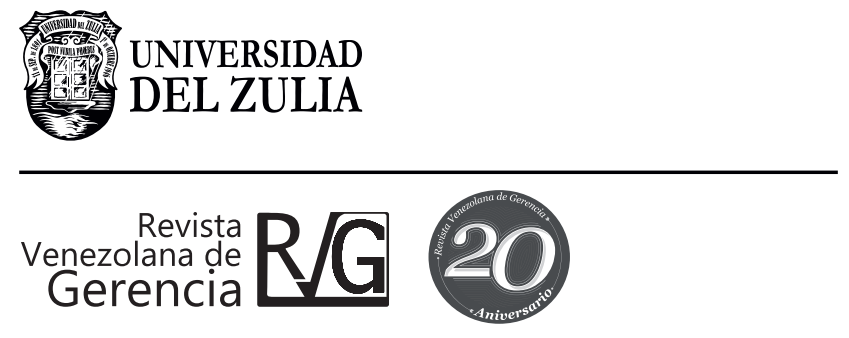

Año 21, No. 74

Esta revista fue editada en formato digital y publicada en junio del 2016, por la Universidad del Zulia, Vicerrectorado Académico, Serbiluz - Fondo editorial, Maracaibo -Venezuela. 